\title{
COMPARISON OF MAGNETIC RESONANCE IMAGING AND CARDIAC CATHETERIZATION IN PATIENTS WITH SUSPECTED SEVERE AORTIC STENOSIS
}

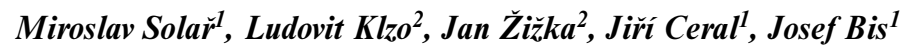

Charles University in Prague, Faculty of Medicine and University Hospital Hradec Králové, Czech Republic: Department of Internal Medicine ${ }^{1}$, Department of Radiology ${ }^{2}$

Summary: Objective. Magnetic resonance imaging (MRI) is a novel technique used in the assessment of aortic stenosis. The aim of the study was to compare MRI and cardiac catheterization (CAT) that is still considered to be a "golden standard" in this indication. Methods. Thirty-four patients referred to CAT for the evaluation of aortic stenosis were enrolled into the study. CAT was performed according to the standardized protocol. Cardiac output was measured by thermodilution and mean aortic gradient was determined using simultaneous blood pressure measurement in aorta and left ventricle. MRI was performed within the period of 3 weeks after CAT. True FISP sequence with retrospective ECG gating was used for the imaging of the aortic valve orifice. Planimetry of the aortic valve area (AVA) was performed at the time of maximal opening of the valve during systole. Results. MRI enabled the measurement of AVA in all patients enrolled. Mean AVA defined by CAT and MRI were $0,97( \pm 0,41) \mathrm{cm}^{2}$ and $1,38( \pm 0,55) \mathrm{cm}^{2}$, respectively. The correlation between the evaluated methods was statistically significant $(\mathrm{p}=0,003)$, but not very strong $(\mathrm{r}=0,43)$. The comparison of both methods in the identification of the severe aortic stenosis was characterized by kappa value of 0,331 . Conclusion. Our study shows low agreement between cardiac catheterization and magnetic resonance imaging in the assessment of aortic stenosis. However, MRI might have a role in the diagnostic algorithm in patients with suspected severe aortic stenosis and moderate mean aortic gradient or concomitant valvular insufficiency.

Key words: Magnetic resonance imaging; Aortic stenosis

\section{Introduction}

Aortic stenosis is the most common valvular heart disease with the estimated prevalence of 1-4\% in adults over 65 years in the developed countries $(11,14)$. The diagnosis and clinical management are usually based upon symptoms, physical examination and measurement of transvalvular gradient by echocardiography (1). However, this approach might be inaccurate in patients with abnormal cardiac output and/or concomitant aortic valve insufficiency $(3,7)$. Some authors therefore consider the determination of aortic valve area (AVA) important for assessing the severity of valvular stenosis.

Although AVA is today most commonly calculated from echocardiographic data (12) using AVA planimetry or continuity equation, the reference technique still remains cardiac catheterization (CAT) as the great portion of AVA related prognostic data were acquired by this method $(13,15)$.

The new diagnostic technique that can be used for the quantification of valvular aortic stenosis is magnetic resonance imaging (MRI) $(2,8)$ that enables an accurate visu- alization of aortic valve orifice. MRI assessment of AVA correlates well with transesophageal echocardiography. However, the published comparisons with cardiac catheterization are equivocal $(4,5,8,10)$.

As the catheterization is routinely used in our center for the assessment the severity of aortic stenosis, we performed this study as to validate MRI against the reference technique.

\section{Methods}

The study enrolled patients who underwent cardiac catheterization for suspected severe aortic stenosis and who agreed with MRI exam. Exclusion criteria were the contraindications of MRI, significant heart rate irregularity (eg. atrial fibrillation) and more than mild aortic insufficiency (>1/5 according to contrast aortography).

\section{Cardiac catheterization}

Cardiac catheterization (CAT) was performed routinely by experienced specialist according to the standardized pro- 
tocol. Retrograde catheterization of the left ventricle was performed with a catheter introduced via a long sheath with its end placed in the ascending aorta. Transseptal approach was chosen when retrograde catheterization of the left ventricle was unsuccessful. This technique enabled simultaneous recording of left ventricular and aortic pressures in all cases. Pressure gradients were calculated from 5-6 cardiac cycles by standard commercial software (Cathcor, Siemens). Cardiac output was measured by thermodilution method using icy saline solution and commercial cardiac output computer (Dualtherm COC, Braun Medical). The mean of three consecutive cardiac output measurements that did not differ by more than $10 \%$ was used for calculation of AVA by Gorlin formula (6).

Contrast ventriculography was used for the calculation of ejection fraction and aortography evaluated the presence of aortic insufficiency.

\section{Magnetic resonance imaging}

MRI was performed within the period of 3 weeks after cardiac catheterization. Before the examination the patients gave written informed consent with the MRI. MR tomograph Siemens Symphony 1,5 T was used for the purposes of this study. Examining specialist was blinded to the results of cardiac catheterization.

True FISP sequence with retrospective ECG gating and voxel size of $1,1 \times 1,3 \times 5 \mathrm{~mm}$ was used for the imaging of the aortic valve. The identification of the optimal slice for the evaluation of the aortic valve was left upon discretion of

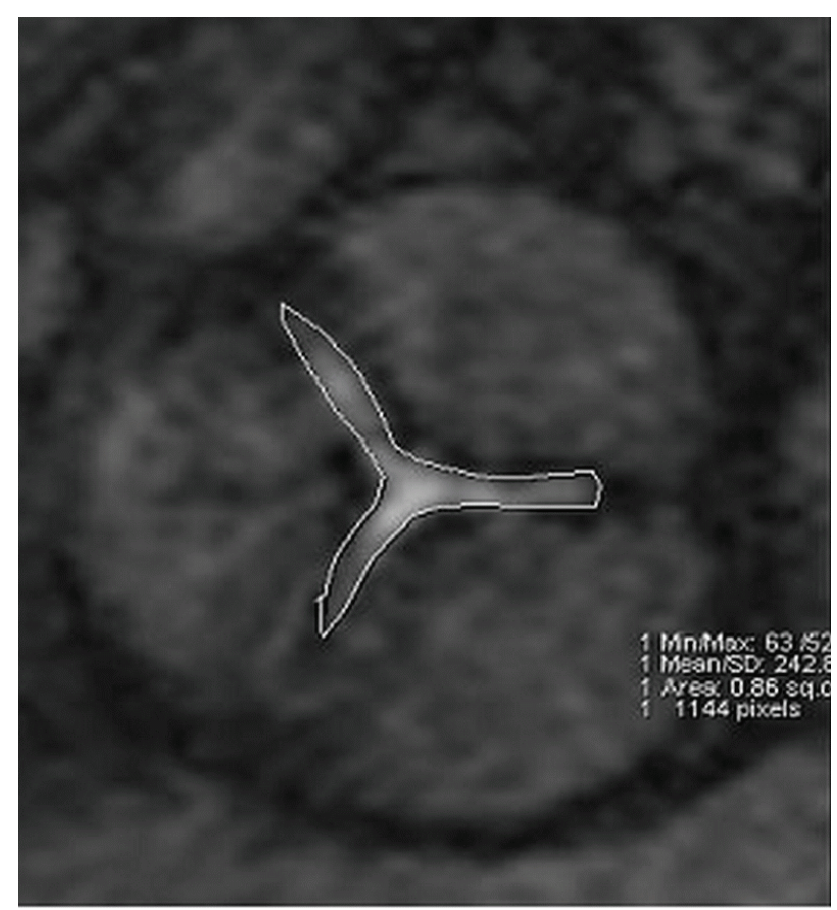

Fig. 1: Magnetic resonance imaging of the aortic valve Planimetry of the aortic valve area was performed at the time of maximal opening of the valve during systole. examining physician. Planimetry of the aortic valve orifice was performed at the time of maximal opening of the valve during systole (Fig. 1). AVA was calculated as the mean of two consecutive planimetric measurements.

\section{Statistical analysis}

Paired t-test, correlation and kappa statistic were used to evaluate an agreement between both methods. Severe aortic stenosis was defined according to ESC guidelines (16), i.e. by ${ }_{\text {indexed }} \mathrm{AVA}<0,6 \mathrm{~cm}^{2} / \mathrm{m}^{2}$.

\section{Results}

Thirty-four patients were enrolled in the study. The principal characteristics of the study population are given in Tab. 1. AVA evaluation by both compared methods was performed within the mean period of $9( \pm 5,6)$ days. Cardiac catheterization was performed according to the standardized protocol in all patients. Retrograde catheterization of the left ventricle was performed in 32 patients and the transseptal approach was chosen in two of them. MRI enabled good visualization of aortic valve in 32 patients. In the remaining two, the imaging quality was poor but the measurement of AVA was possible. Both patients with poor imaging quality had massive valve calcification and marked in plane valve motion. The data from cardiac catheterization and magnetic resonance imaging are summarized in Tab. 2.

Tab. 1: Principal characteristics of study population.

\begin{tabular}{|l|c|}
\hline Number of patients (women) & $34(9)$ \\
\hline Age (years) & $64( \pm 14,1)$ \\
\hline History & $10(29,4 \%)$ \\
Coronary artery disease & $12(35,3 \%)$ \\
Diabetes mellitus & $16(47,1 \%)$ \\
Arterial hypertension & $14(41,2 \%)$ \\
Hyperlipidemia & \\
\hline
\end{tabular}

Table 1 shows the principal characteristics of study population. Values are expressed as number of patients $(\%)$ or mean $( \pm$ standard deviation).

Tab. 2: Data from cardiac catheterization and magnetic resonance imaging.

\begin{tabular}{|l|c|}
\hline Ejection fraction $(\%)$ & $59( \pm 15,7)$ \\
\hline Cardiac output $(1 / \mathrm{min})$ & $4,9( \pm 1,54)$ \\
\hline Cardiac index $\left(1 / \mathrm{min} / \mathrm{m}^{2}\right)$ & $2,5( \pm 0,77)$ \\
\hline Mean aortic gradient $(\mathrm{mm} \mathrm{Hg})$ & $42( \pm 23,1)$ \\
\hline Peak to peak aortic gradient $(\mathrm{mm} \mathrm{Hg})$ & $50( \pm 31,7)$ \\
\hline CAT AVA $\left(\mathrm{cm}^{2}\right)$ & $1,0( \pm 0,41)$ \\
\hline CAT indexed AVA $\left(\mathrm{cm}^{2} / \mathrm{m}^{2}\right)$ & $0,5( \pm 0,19)$ \\
\hline Aortic insufficiency $\left(\mathrm{grade}^{2} / 5\right)$ & $20(61 \%)$ \\
\hline MRI AVA $\left(\mathrm{cm}^{2}\right)$ & $1,3( \pm 0,54)$ \\
\hline MRI indexed AVA $\left(\mathrm{cm}^{2} / \mathrm{m}^{2}\right)$ & $0,7( \pm 0,26)$ \\
\hline
\end{tabular}

Table 2 summarizes data from cardiac catheterization (CAT) and magnetic resonance imaging (MRI). Values are expressed as mean ( \pm standard deviation) or number of patients $(\%)$. 
Tab. 3: Cardiac catheterization and MRI in the detection of severe aortic stenosis.

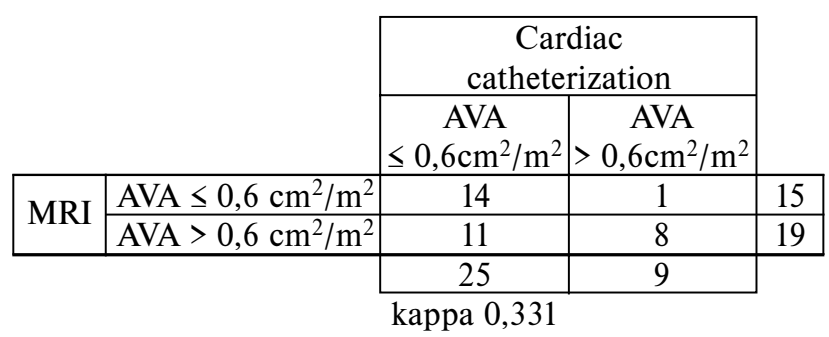

Table 3 shows pairwise comparison of cardiac catheterization and magnetic resonance imaging (MRI) in the diagnosis of severe aortic stenosis (AVA $\leq 0,6 \mathrm{~cm}^{2} / \mathrm{m}^{2}$ ). Kappa value 0,331 reflects fair agreement between both methods.

Graph 1 shows comparison of mean AVAs determined by both methods. Magnetic resonance imaging significantly overestimated aortic valve area compared with cardiac catheterization.

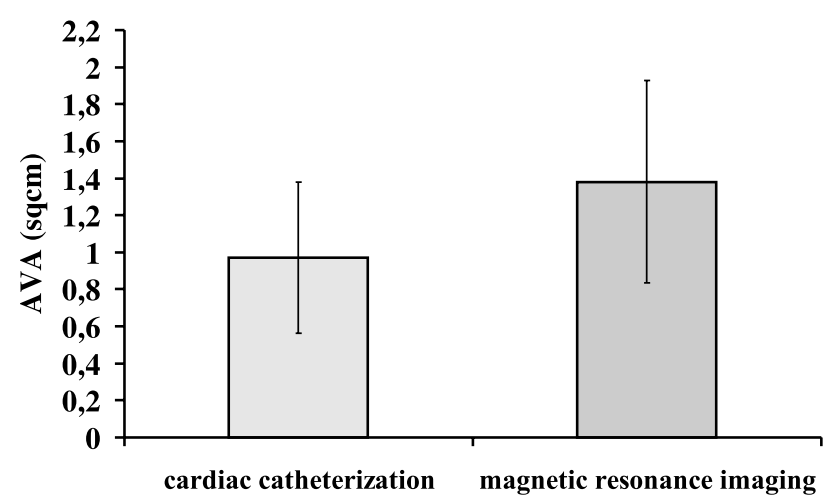

Graph 1: Mean aortic valve area determined by cardiac catheterization and magnetic resonance imaging.

Graph 2 shows the correlation between cardiac catheterization (CAT) and magnetic resonance imaging (MRI) in the assessment of indexed aortic valve area (AVA). The correlation was statistically significant $(p=0,003)$ but not very strong $(r=0,43)$.

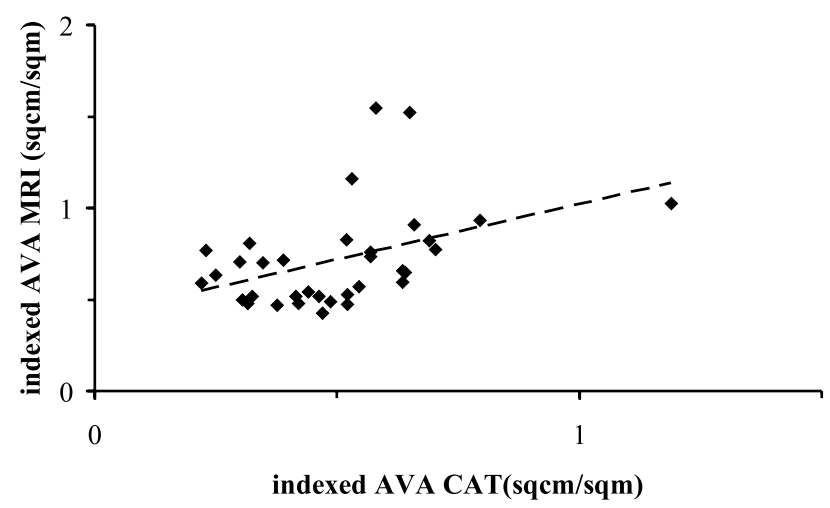

Graph 2: The correlation between cardiac catheterization and magnetic resonance imaging in the evaluation of aortic valve area.

\section{Comparison of AVA defined by both methods}

Mean AVA defined by catheterization was $0,97( \pm 0,41)$ $\mathrm{cm}^{2}$ and mean AVA calculated from MRI was $1,38( \pm 0,55)$ $\mathrm{cm}^{2}$ (Graph 1). The overestimation of aortic valve orifice by MRI was statistically significant $(p<0,001)$. The mean difference in indexed AVA determined by MRI and CAT was $0,22 \mathrm{~cm}^{2} / \mathrm{m}^{2}$ (range $0,16-0,96$ ).

The correlation of indexed AVAs calculated by both methods (Graph 2) was statistically significant $(\mathrm{p}=0,003)$, but not very strong $(\mathrm{r}=0,43)$. Better results were not observed when the analysis was limited to the patients with cardiac index $>2,41 / \mathrm{m}^{2}$ and/or without detectable aortic insufficiency. The comparison of CAT and MRI in the identification of severe aortic stenosis is shown in Tab. 3 . Kappa value 0,331 reflects fair agreement only.

\section{Discussion}

MRI enabled good visualization of aortic valve in the great majority examined patients. The imaging quality was worse in individuals with massive valve calcifications and in-plane valve motion, but the AVA measurement was possible in all patients enrolled.

We did not prove an agreement between cardiac catheterization and magnetic resonance imaging in the evaluation of aortic stenosis. In accordance with data published by Debl (4), our study demonstrated an overestimation of AVA by MRI.

Provided the cardiac catheterization was considered to be the reference technique, we were not able to find a valid cut off value for indexed AVA determined by MRI that would be characterized by acceptable sensitivity and specificity for the diagnosis of severe aortic stenosis. However, high specificity (93\%) of MRI was observed in patients where MRI described severe aortic stenosis $\left({ }_{\text {indexed }}\right.$ AVA $<0,6 \mathrm{~cm}^{2} / \mathrm{m}^{2}$ ).

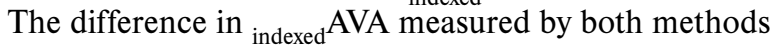
was significant and led to low level of agreement in the identification of severe aortic stenosis. Eleven out of 25 patients with CAT defined severe aortic stenosis did not fulfill the criteria for severity according to MRI study. In six of them the mean aortic gradient was $<50 \mathrm{~mm} \mathrm{Hg}$ (range 15-39 $\mathrm{mm} \mathrm{Hg}$ ), not fulfilling the echo Doppler criteria defining the severe aortic stenosis. In five patients with mean aortic gradient $>50 \mathrm{~mm} \mathrm{Hg}$, no elevation in cardiac index was noted. As to better understand the observed discrepancies we describe two illustrative cases:

In patient 1 the difference between both methods was

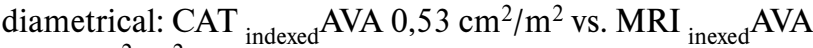
$1,13 \mathrm{~cm}^{2} / \mathrm{m}^{2}$. The mean aortic gradient was low (15 $\mathrm{mmHg}$ ). Because of this discrepancy we performed a new MRI, but the repeated, unblinded exam failed to find any significant narrowing of the aortic valve. Based on this finding the patient was left on medical therapy and was not referred to the aortic valve replacement.

In patient 2 the catheterization confirmed severe aortic stenosis: mean aortic gradient $96 \mathrm{~mm} \mathrm{Hg}$ and cardiac index 


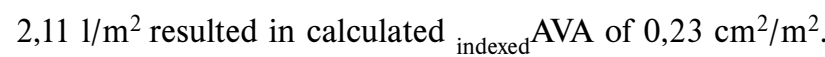
On the other hand MRI showed only moderate aortic stenosis with indexed AVA $0,77 \mathrm{~cm}^{2} / \mathrm{m}^{2}$. As the high mean aortic gradient was also found at echocardiography and the patient was symptomatic, aortic valve replacement was performed.

In our opinion, both compared techniques have inherent limitations and none of them is principally ideal. Concerning the cardiac catheterization, in comparison to other studies $(8,10)$ we used more precise method for AVA determination. Together with excluding patients with irregular heart rate and more than minimal aortic insufficiency, simultaneous measurement of aortic and left ventricular pressures and the determination of cardiac output by thermodilution were used. Although the latter has its inherent limitations we assume it to be more precious than using estimated oxygen consumption data. Furthermore, it is not clear whether the constant of Gorlin formula is valid for various shapes of aortic valves and low cardiac output (17).

Concerning the MRI, the images are obtained from a section of a $5 \mathrm{~mm}$ thickness that is moving during the data acquisition. The planar MRI images need not completely correspond to the three-dimensional shape of the imaged object and to the real resistance of the stenotic valve. Inaccuracies in measuring AVA can be also attributed to flow turbulence and calcifications that are often present in severe valvular lesions.

\section{Conclusion}

Our study shows low agreement between cardiac catheterization and magnetic resonance imaging in the assessment of aortic stenosis. Magnetic resonance significantly overestimates the size of aortic valve orifice. Based on our data we were not able to determine MRI indexed AVA cut off that would reliably identify severe aortic stenosis as defined by cardiac catheterization. However, MRI might have a role in the diagnostic algorithm in patients with suspected severe aortic stenosis and mild to moderate mean aortic gradient or concomitant aortic insufficiency. MRI results seem to be valid when severe valvular narrowing is found. These pa- tients might be consequently "saved from" the need of invasive hemodynamic study. On the other hand, cardiac catheterization is definitely indicated when the aortic stenosis is described as moderate according to MRI.

\section{References}

1. Bonow RO, Carabello B, de LeonJr. AC et al. ACC/AHA guidelines for the management of patients with valvular heart disease: A report of the American College of Cardiology/American Heart Association Task Force on practice guidelines (Committee on management of patients with valvular heart disease). J Am Coll Cardiol 1998; 32(5):1486-582.

2. Caruthers SD, Lin SJ, Brown P et al. Practical Value of Cardiac Magnetic Resonance Imaging for Clinical Quantification of Aortic Valve Stenosis: Comparison With Echocardiography. Circulation 2003; 108(18):2236-43.

3. Danielsen R, Nordrehaug JE, Stangeland L, Vik-Mo H. Limitations in assessing the severity of aortic stenosis by Doppler gradients. Br Heart J 1988; 59(5):551-5.

4. Debl K, Djavidani B, Seitz J et al. Planimetry of aortic valve area in aortic stenosis by magnetic resonance imaging. Invest Radiol 2005; 40(10):631-6.

5. Friedrich MG, Schulz-Menger J, Poetsch T, Pilz B, Uhlich F, Dietz R Quantification of valvular aortic stenosis by magnetic resonance imaging. Am Heart J 2002; 144(2):329-34.

6. GORLIN R, GORLIN SG. Hydraulic formula for calculation of the area of the stenotic mitral valve, other cardiac valves, and central circulatory shunts. I. Am Heart J 1951; 41(1):1-29.

7. Griffith MJ, Carey C, Coltart DJ, Jenkins BS, Webb-Peploe MM. Inaccuracies in using aortic valve gradients alone to grade severity of aortic stenosis. Br Heart J 1989; 62(5):372-8.

8. John AS, Dill T, Brandt RR et al. Magnetic resonance to assess the aortic valve area in aortic stenosis: How does it compare to current diagnostic standards? J Am Coll Cardiol 2003; 42(3):519-26.

9. Kendrick AH, West J, Papouchado M, Rozkovec A. Direct Fick cardiac output: are assumed values of oxygen consumption acceptable? Eur Heart J 1988; 9(3):337-42.

10. Kupfahl C, Honold M, Meinhardt G et al. Evaluation of aortic stenosis by cardiovascular magnetic resonance imaging: comparison with established routine clinical techniques. Heart 2004; 90(8):893-901.

11. Lindroos M, Kupari M, Heikkila J, Tilvis R. Prevalence of aortic valve abnormalities in the elderly: an echocardiographic study of a random population sample. J Am Coll Cardiol 1993; 21(5):1220-5.

12. Mochizuki Y, Pandian NG. Role of echocardiography in the diagnosis and treatment of patients with aortic stenosis. Curr Opin Cardiol 2003; 18(5):327-33.

13. Rahimtoola S. Perspective on valvular heart disease: an update. J Am Coll Cardiol 1989; 14(1):1-23

14. Stewart BF, Siscovick D, Lind BK et al. Clinical factors associated with calcific aortic valve disease. Cardiovascular Health Study. J Am Coll Cardiol 1997; 29(3):630-4

15. Turina J, Hess O, Sepulcri F, Krayenbuehl H. Spontaneous course of aortic valve disease. Eur Heart J 1987; 8(5):471-83.

16. Vahanian A, Baumgartner H, Bax J et al. Guidelines on the management of valvular heart disease: The Task Force on the Management of Valvular Heart Disease of the European Society of Cardiology. Eur Heart J 2007: 28(2):230-68.

17. Weyman AE, Scherrer-Crosbie M. Aortic stenosis: physics and physiology-what do the numbers really mean? Rev Cardiovasc Med 2005; 6(1):23-32.

Submitted April 2008 Accepted June 2008.

Corresponding author:

Miroslav Solař, M.D., Departement of Internal Medicine, University Hospital Hradec Králové, Sokolská 581, 50005 Hradec Králové, Czech Republic; e-mail: solarmir@seznam.cz 